\title{
The 3-inch Photomultiplier System of the JUNO Experiment
}

\section{Cécile Jollet ${ }^{a, *}$ on behalf of the JUNO collaboration}

${ }^{a}$ Univ. Bordeaux, CNRS, CENBG, UMR 5797, F-33170 Gradignan, France

E-mail: cecile.jollet@cenbg.in2p3.fr

JUNO is a multipurpose experiment under construction in China. In addition to 18000 20-inch large photomultipliers (LPMTs) serving as the primary light-detection device, the JUNO central detector will be instrumented with 25600 custom-made 3 -inch small photomultipliers (SPMTs) to boost the physics potential of the experiment. The SPMTs will mostly operate in the photoncounting and will help to calibrate the energy response of the LPMTs. They will also aid the measurement of supernova neutrinos and will improve the muon track reconstruction whose performance is important for background rejection. The SPMTs, together with their power and readout systems, will have to operate under water for over 20 years, posing challenging constraints on the design, reliability and implementation of this major subsystem of JUNO. In this poster, we will present the innovative design of the JUNO SPMT system, its impact on physics, and the current status of SPMT production and testing.

40th International Conference on High Energy physics - ICHEP2020

July 28 - August 6, 2020

Prague, Czech Republic (virtual meeting)

\footnotetext{
${ }^{*}$ Speaker
} 


\section{Introduction}

JUNO (Jiangmen Underground Neutrino Observatory) is a 20 kton liquid scintillator detector aiming at the observation of the $\overline{v_{e}} \rightarrow \overline{v_{e}}$ transition exploting reactor antineutrinos emitted from the Yangjiang and Taishan reactor complexes $53 \mathrm{~km}$ away. The main physics goals are the determination of the neutrino mass hierarchy and the sub-percent measurement of the solar oscillation parameters $\Delta m_{21}^{2}$ and $\sin ^{2} \theta_{12}$ ( [1] and [2]).

The energy resolution is critical to perform the mass hierarchy measurement and the goal is to achieve $3 \%$ at $1 \mathrm{MeV}$, which means that about 1200 photoelectrons should be collected. A large photo-coverage is therefore needed and 18000 20-inch PMTs and 25600 3-inch PMTs will be installed in the JUNO detector as shown in Fig. 1.

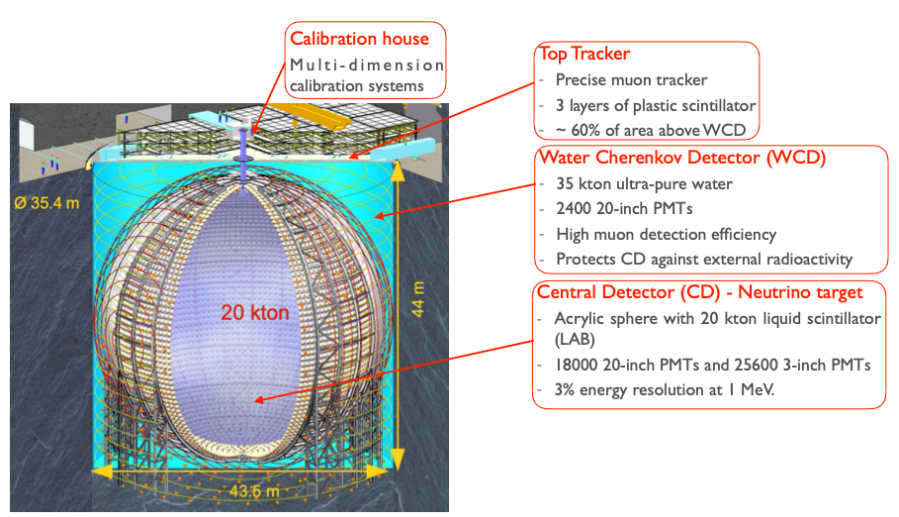

Figure 1: Schematic view of the JUNO detector.

\section{Small PMT system}

The SPMTs, from HZC photonics [3] will be integrated in JUNO interleaving the two type of PMTs reducing as much as possible the dead space as shown in Fig. 2(a). If the total photocoverage is increased by on $3 \%$, the complementary photo-detection system presents several specific advantages for the JUNO experiment.

- It provides a complementary and independent read-out.

- It helps to reach a precision calorimetry setting the energy scale given the different saturation level with respect to 20-inch PMTs.

- It improves the muon track reconstruction resolution since SPMTs are not saturated by the muon energy deposits.

- It improves the detector particle identification.

- It can detect spallation neutrons since the dead time after muons is very small.

- It provides an additional measurement of the so-called solar oscillation parameters.

- It can measure supernova events standing a high event rate. 


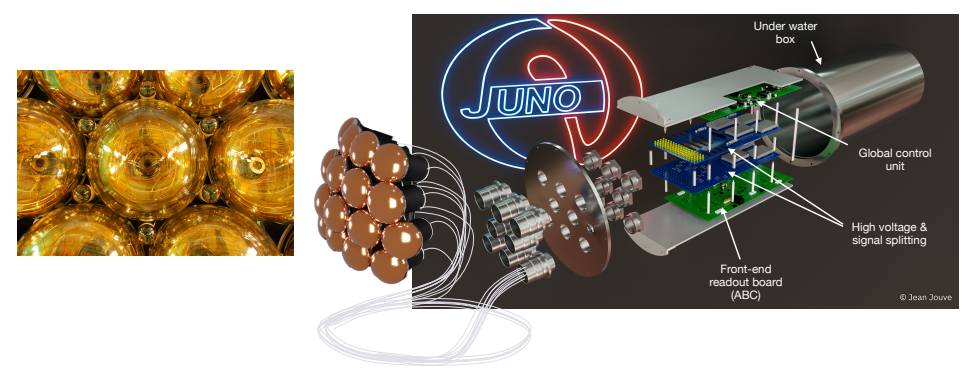

(a)

(b)

Figure 2: 20-inch and 3-inch PMT interleaving (a), and cartoon showing the SPMTs system and its electronics $(b)$.

These performances can be reached thanks to the very good transit time (1.6 ns) and the fact that the mean illumination of the SPMTs is low enough for them to be always working in photo-counting regime. Specific underwater cables were developed in collaboration with Axon [4] company and all the electronics for a group of 128 PMTs will be hosted under water boxes which can hold up to 8 bar pressure. The front-end readout board is custom made, conceived and produced by IN2P3 groups in France and based on the CATIROC readout ASIC produced by Omega [5]. A schematic cartoon of the whole system can be seen in Fig. 2(b) and test to validate the full chain are smoothly ongoing.

\section{Conclusions}

To determine the mass hierarchy in JUNO the energy resolution is a key ingredient and the SPMT system will be an important handle to meet the requirements. In addition this independent light readout system will play a key role in several background measurements and physics analyses thanks to the fact that SPMTs are not saturated by events releasing a large amount of energy inside the detector.

The 25600 PMTs have ben produced and a full chain including 128 PMTs has been successfully tested. The system is expected to be ready in 2021, for a start of the data taking with the full detector in 2022 .

\section{References}

[1] Z. Djurcic et al. [JUNO], [arXiv:1508.07166 [physics.ins-det]].

[2] F. An et al. [JUNO], J. Phys. G 43, no.3, 030401 (2016).

[3] http://www.hzcphotonics.com/products/XP72B20.pdf

[4] https://www.axon-cable.com/fr/00_accueil/00_sommaire/00/index.aspx

[5] S. Conforti et al. Springer Proc. Phys. 212, 168-172 (2018) 